\title{
Leiomyosarcomas and Most Malignant Fibrous Histiocytomas Share Very Similar Comparative Genomic Hybridization Imbalances: An Analysis of a Series of 27 Leiomyosarcomas
}

\author{
Josette Derré, Réal Lagacé, André Nicolas, Aline Mairal, Frédéric Chibon, \\ Jean-Michel Coindre, Philippe Terrier, Xavier Sastre, and Alain Aurias \\ Laboratoire de Pathologie Moléculaire des Cancers, INSERM U509, Institut Curie (JD, AM, FC, AA), Paris; the \\ Departments of Pathology from Institut Curie (AN, XS), Paris, Institut Bergonié (JMC), Bordeaux, and Institut \\ Gustave Roussy (PT), Villejuif, France; and the Department of Pathology from Centre Hospitalier Universitaire de \\ Québec-Hôtel Dieu de Québec (RL), Canada
}

\begin{abstract}
SUMMARY: Twenty-seven tumor samples with a diagnosis of leiomyosarcomas (LMS) were characterized by comparative genomic hybridization. The results were compared with immunohistochemical analysis of the smooth muscle profile of the tumors and expression of the RB1 gene protein. The comparative genomic hybridization profiles suggested that 7 of the 27 tumors might have been misclassified. High levels of DNA amplification were detected in 20 different small regions and recurrently involved bands 1p34, 1q21, 12q13-15, 17p, and 22q. Most recurrent simple gains were noted at sites such as 1p3, 1q21, 15q12-15, 16p, $17 p$ and $17 q, 19,20 q, 22 q$, and Xp. Significant losses of chromosome 13 were detected in 19 of the 27 tumors with a putative common region of loss in bands 13q14-21. Losses of chromosomes 1q, 2p and 2q, 4q, 9p, 10p and 10q, 11p and 11q23, and $16 q$ were also highly recurrent. A comparative analysis between the most frequent genomic imbalances observed in this study of LMS and the genomic imbalances observed in a large proportion of malignant fibrous histiocytomas (MFH) from a previous study demonstrated that both types of tumors had similar recurrent imbalances. Although MFH were once thought to be a separate member of the soft tissue sarcoma family, our observations support the hypothesis that MFH are a morphologic modulation in the tumoral progression of other sarcomas, particularly LMS. (Lab Invest 2001, 81:211-215).
\end{abstract}

\begin{abstract}
Ciomyosarcomas (LMS) are relatively frequent sar$L_{\text {comas of adult life. They are principally tumors of }}$ uterine or gastrointestinal origin, but also account for $5 \%$ to $10 \%$ of soft tissue sarcomas (STS) (Enzinger and Weiss, 1995). Within the past few years, with better identification of dedifferentiated liposarcomas and the recognition of malignant fibrous histiocytomas (MFH) as a separate entity, the relative incidence of LMS among STS has changed. The recent characterization of gastrointestinal stromal tumors (GIST) as a distinct classification also influences the incidence of gastrointestinal and retroperitoneal LMS. Contrary to $\mathrm{MFH}$, osteosarcomas, or fibrosarcomas, LMS rarely occur after radiation, but may develop in patients with a constitutional inactivation of one allele of the RB1 gene and with a previous diagnosis of bilateral retinoblastoma. LMS are much more common in women
\end{abstract}

Received November 2, 2000.

Supported by grants from INSERM, ARC, the Ligue Nationale Contre le Cancer (Comité de Paris and Comité de l'Oise), and the FEGEFLUC.

Address reprint requests to: Dr. Alain Aurias, Laboratoire de Pathologie Moléculaire des Cancers, INSERM U509, Institut Curie, Section de Recherche, 26 rue d'Ulm, 75248 Paris cedex 05, France. E-mail: alain.aurias@curie.fr than men, even for nonuterine locations. Until now, few cytogenetic or molecular data were available for LMS (Mandahl et al, 2000), and only a few series were analyzed by comparative genomic hybridization (CGH) (El-Rifai et al, 1998; Otano-Joos et al, 1998; Packenham et al, 1997; Parente et al, 1999). In this study, we analyzed a series of 27 LMS by immunohistochemistry and $\mathrm{CGH}$. We found that the most frequent genomic alterations in LMS were losses in the 13q14-21 region. LMS and a large proportion of MFH were also found to share very similar CGH imbalance profiles, which suggests that these two tumors may correspond to different differentiation states of a single tumor type.

\section{Results}

\section{Imbalances Detected by CGH}

One of the 27 samples (LMS 23) did not exhibit any imbalance. A considerable contamination of this tumor sample by nontumoral cells might account for these findings. Another tumor (LMS 28) had a single aberration, which was not recurrently observed in the other samples. The regions of DNA copy number 
imbalances observed in the 27 tumors are shown in Figure 1.

Gains of DNA. High levels of DNA amplification (green to red ratio greater than 1.5) were observed in 20 different small regions. Among them, bands 1p34, $1 q 21,12 q 13-15,17 p$, and $22 q$ were recurrently observed as amplified regions in different tumors. The most recurrent simple gains involved 1p3, 1q21, 15q12-15, 16p, 17p and 17q, 19, 20q, 22q, and Xp.

Losses of DNA. As shown in Figure 1, significant losses in chromosome 13 were observed in 19 of the 27 tumors, with a putative common region of loss in bands 13q14-21. In one other tumor (LMS 7), probably contaminated by a large number of normal cells, chromosome 13 loss was suggested by a green to red ratio of 0.85 . Losses of chromosome $1 q, 2 p$ and $2 q$, $4 q, 9 p, 10 p$ and $10 q, 11 p$ and $11 q 23$, and $16 q$ were also recurrent.

\section{Immunohistochemistry}

Results are summarized in Table 1 along with the main clinical data. In general, expression of the smooth muscle markers, common muscle actin (HHF 35), smooth muscle actin, and desmin, did not strictly correlate with tumor grade. Some low-grade LMS were negative for desmin expression, whereas some high-grade LMS were strongly immunoreactive for the three smooth muscle markers. There was no correlation between grade, intensity of immunoreactivity of the smooth muscle markers, and expression of Rb1 gene protein. With one exception, all tumors were
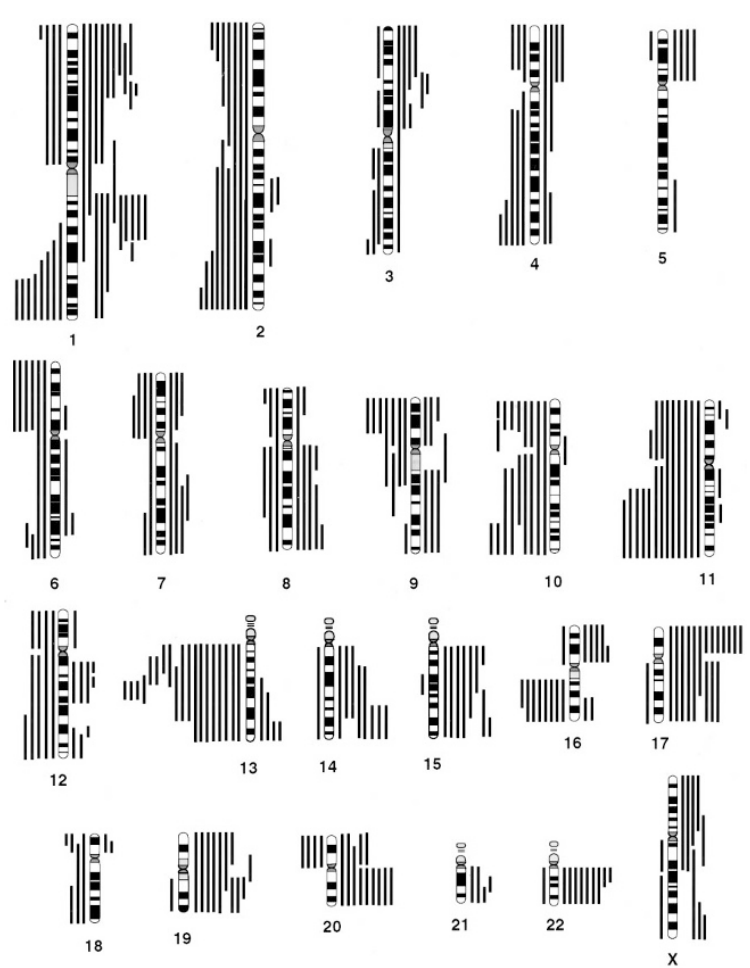

Figure 1.

Ideogram of regions of DNA copy number imbalances. Vertical lines on the right and left sides of chromosomes indicate gains and losses, respectively. negative for CD 34 and CD 117. The one exception (LMS 14) originated in the retroperitoneum, was of a large volume (15 cm in diameter), and was immunoreactive for common muscle actin (HHF 35) and smooth muscle actin.

\section{Discussion}

The CGH analysis of a series of 27 LMS showed the occurrence of genomic imbalances in all tumors but one. In this tumor, LMS 23, we did not find either chromosome imbalance by CGH or allelic imbalance by microsatellite allelotyping (data not shown). The presence of a prominent inflammatory component in this tumor could account for the normal CGH profiles. In LMS 28, the only detected imbalance corresponded to an intercalary deletion of the 9q21-31 bands. At the time of diagnosis, the patient had two synchronous tumors; one tumor within the transverse colon ( $4 \mathrm{~cm}$ in diameter) and a second tumor within the pelvis (12 cm in diameter). This patient is alive and free of disease 5 years later, which raises the possibility that both synchronous low grade LMS were in fact multiple leiomyomas. Three unique tumors from the abdominal cavity, LMS 43, LMS 45, and LMS 20, expressed the three muscular markers and did not express CD34 or CD117. All three tumors had losses of whole 1p; LMS 43 also had a gain of whole 1q and LMS 45 also had a loss of chromosome 14. These profiles are suggestive of GIST (Breiner et al, 2000; Derré et al, 2000; El-Rifai et al, 1996, 2000). Moreover, a number of GIST can express muscular markers (Miettinen et al, 1999; Sircar et al, 1999). Three other tumors (LMS 40, LMS 21, and LMS 47) were characterized by simple CGH profiles associated with multiple small amplifications (such as bands 12q14-15). This is reminiscent of the profiles found in well-differentiated liposarcomas (Suijkerbuijk et al, 1994). The morphologic characterization of LMS 14 was difficult to assess because two muscular markers (HHF35 and smooth muscle actin) were expressed along with moderate CD 117 expression, which could be suggestive of GIST, but the CGH profile of LMS 14 was incompatible with a GIST diagnosis. Thus, the $\mathrm{CGH}$ analysis of this series of 27 tumors suggests that 7 of them might have been misclassified by histopathologic and immunohistochemistry criteria alone.

We did not find clear correlations between the sizes of the tumors, grades, and any of the aberrations observed. In particular, gains of 16p, 17p, 1q, and 8q, previously reported as correlating with size (El-Rifai et al, 1998), seemed relatively widespread in the tumors analyzed in our study. Our immunohistochemical findings did not support this correlation.

As previously noted in a small series of LMS (OtanoJoos et al, 1998), chromosome 13 loss, observed in 19 of the tumors in our series, was the most frequent genomic aberration. In the current study, CGH demonstrated that the smallest region of overlap of these losses was localized to the $13 q 14-21$ region. As recently demonstrated for MFH (Chibon et al, 2000), these observations could suggest that the RB1 gene is also the target of these deletions. Our immunohistochemical analysis, which showed that most tumors 
Table 1. Results of Immunohistochemistry

\begin{tabular}{|c|c|c|c|c|c|c|c|c|c|c|c|c|c|c|c|}
\hline Cases & Sex & Age & Grade & $\mathrm{PT} / \mathrm{R}$ & $\mathrm{Tt}$ & Site & Size & HHF35 & Sarc & Smooth & Desm & Vim & CD34 & CD117 & RB1 \\
\hline LMS 05 & $M$ & 75 & II & $\mathrm{R}$ & $\mathrm{RT}$ & Back & 3 & 0 & 0 & 0 & + & +++ & 0 & 0 & 0 \\
\hline LMS 07 & $\mathrm{~F}$ & 88 & III & PT & & Breast & 8 & + & 0 & 0 to + & 0 & +++ & 0 & 0 & + \\
\hline LMS 10 & $\mathrm{~F}$ & 60 & III & PT & CT & Breast & 9 & ++ & + & + & ++ & ++ & 0 & 0 & $\begin{array}{l}+ \text { to } \\
++\end{array}$ \\
\hline LMS 11 & $\mathrm{~F}$ & 64 & III & PT & CT & Thigh & 13 & 0 & 0 & + & 0 & +++ & 0 & 0 & 0 \\
\hline LMS 12 & M & 59 & I & PT & & Arm & 3 & +++ & 0 & +++ & 0 & 0 to + & 0 & 0 & 0 to + \\
\hline LMS 14 & M & 49 & III & PT & & Retrop & 15 & ++ & 0 & ++ & 0 & 0 to + & +++ & $\begin{array}{l}+ \text { to } \\
++\end{array}$ & 0 \\
\hline LMS 17 & $\mathrm{~F}$ & 41 & II & PT & & Breast & 3 & 0 & 0 & 0 & ++ & +++ & 0 & 0 & + \\
\hline LMS 19 & $M$ & 64 & III & PT & CT & Neck & 10 & ++ & 0 & + & 0 & +++ & 0 & 0 & 0 to + \\
\hline LMS 20 & $\mathrm{~F}$ & 64 & III & PT & & Small int & 10 & +++ & 0 & +++ & +++ & +++ & 0 & 0 & 0 to + \\
\hline LMS 21 & $M$ & 57 & III & PT & & Retrop & 10 & + & 0 & ++ & 0 & +++ & 0 & 0 & ++ \\
\hline LMS 22 & $\mathrm{~F}$ & 42 & III & PT & $\mathrm{RT}$ & Pelvis & 11 & + & ++ & 0 & + & +++ & 0 & 0 & 0 to + \\
\hline LMS 23 & $\mathrm{~F}$ & 52 & III & PT & & Trunk & 11 & + & 0 & ++ & 0 & +++ & 0 & 0 & ++ \\
\hline LMS 24 & $\mathrm{~F}$ & 49 & III & PT & & Retrop & 17 & +++ & 0 & +++ & +++ & +++ & 0 & 0 & 0 \\
\hline LMS 25 & $\mathrm{~F}$ & 70 & I & PT & & Leg & 10 & +++ & 0 & +++ & +++ & ++ & 0 & 0 & 0 to + \\
\hline LMS 26 & $F$ & 50 & III & PT & & Trunk & 15 & +++ & 0 & +++ & ++ & ++ & 0 & 0 & 0 to + \\
\hline LMS 28 & M & 24 & I & PT & & Colon & 11 & +++ & 0 & +++ & +++ & ++ & 0 & 0 & 0 to + \\
\hline LMS 29 & $F$ & 44 & III & PT & & Pelvis & 10 & +++ & 0 & +++ & +++ & +++ & 0 & 0 & 0 to + \\
\hline LMS 30 & $\mathrm{~F}$ & 67 & III & PT & & Cervix & 11 & 0 & 0 & + & 0 & +++ & 0 & 0 & 0 to + \\
\hline LMS 31 & $\mathrm{~F}$ & 36 & II & PT & & Uterus & 10 & ++ & 0 & ++ & $\begin{array}{c}0 \text { to } \\
+\end{array}$ & +++ & 0 & 0 & $\begin{array}{l}0 \text { to } \\
++\end{array}$ \\
\hline LMS 32 & $\mathrm{~F}$ & 75 & II & PT & & Groin & 3 & +++ & 0 & +++ & $\begin{array}{c}0 \text { to } \\
+\end{array}$ & ++ & 0 & 0 & 0 \\
\hline LMS 36 & M & 26 & II & PT & CT & Femur & 5 & + & 0 & + & +++ & +++ & 0 & 0 & + \\
\hline LMS 38 & $\mathrm{~F}$ & 73 & I & PT & & V. cava & 5 & +++ & 0 & +++ & +++ & 0 to + & 0 & 0 & + \\
\hline LMS 40 & M & 64 & III & PT & & Thigh & 8 & ++ & 0 & ++ & ++ & +++ & 0 & 0 & $\begin{array}{l}+ \text { to } \\
++\end{array}$ \\
\hline LMS 41 & $F$ & 52 & II & PT & & Iliac & 2 & +++ & + & +++ & +++ & +++ & 0 & 0 & $\begin{array}{l}+ \text { to } \\
++\end{array}$ \\
\hline LMS 43 & $\mathrm{~F}$ & 65 & I & $\mathrm{R}$ & & Pelvis & 13 & +++ & 0 & +++ & +++ & ++ & 0 & 0 & $\begin{array}{l}0 \text { to } \\
++\end{array}$ \\
\hline LMS 45 & $\mathrm{~F}$ & 56 & I & PT & & Retrop & 10 & ++ & U & ++ & ++ & + & 0 & 0 & 0 to + \\
\hline LMS 47 & $M$ & 61 & III & PT & & Stom & 9 & ++ & 0 & ++ & ++ & ++ & 0 & 0 & 0 to + \\
\hline
\end{tabular}

LMS, leiomyosarcoma; PT, primary tumor; R, relapse; Tt, treatment; RT, radiotherapy; CT, chemotherapy; Sarc, sarcomeric; Desm, desmin; Vim, vimentin; F, female; M, male.

with chromosome 13 deletions did not express the RB1 protein, supports this hypothesis. Our data is also in agreement with the known occurrence of LMS in patients with bilateral retinoblastoma, who have constitutional inactivation of one allele of RB1 (Dunkel et al, 1998).

Apart from the chromosome 13 deletion, which is common to most tumors, our CGH results are similar to those observed in approximately $80 \%$ of MFH (Mairal et al, 1999), particularly the results of tumors that seem correctly diagnosed. A comparative analysis between the most frequent genomic imbalances observed in the present series of LMS and those of our previous findings on MFH (Mairal et al, 1999; and unpublished data) are presented in Figure 2. Almost all recurrent imbalances are observed in both series, especially the most frequently recurring imbalances, which are thought to correspond to precocious oncogenic events. The complexity of the $\mathrm{CGH}$ profiles and the fact that similar profiles have not been described in other malignancies (for review, see Knuutila et al: CGH Data of DNA Sequence
Number Changes in Human Neoplasms, http:// www.helsinki.fi/lgl_www/CMG.html), strongly suggest that the profiles are not similar by chance. The morphologic studies to characterize MFH were not able to delineate specific markers or to describe the phenotype for this sarcoma of supposed "fibrohistiocytic" lineage. There is growing evidence that MFH is a second component in another sarcoma and represents a morphologic modulation resulting from tumor progression (Dehner, 1988; Fletcher, 1987; Hollowood and Fletcher, 1995). The five previously described subtypes of MFH seem to have few links, if any, to tumors of fibrohistiocytic differentiation, and the angiomatoid variant is no longer thought to be a fully sarcomatous member of STS (Costa and Weiss, 1990; Fanburg-Smith and Miettinen, 1999). Rather, the giant cell variant shares characteristics common to giant-cell-rich osteosarcoma of soft tissues (Guccion and Enzinger, 1972) and a number of inflammatory MFH overlap with inflammatory LMS (Merchant et al, 1995). Additionally, poorly differentiated myxoid MFH merge with pleomorphic MFH (Weiss and 
Enzinger, 1977), which are no longer thought to be tumors of true histiocytic differentiation. Our results suggest that MFH do not represent a specific sarcomatous entity, but may instead represent a common oncogenic pathway in the progression of LMS and possibly other STS. If this hypothesis is correct, most MFH could represent an ultimate state of tumor progression for LMS. Nevertheless, among the 26 imbalances listed in Figure 2, $6(+14,+15 q,+18 p,-6 p,+6 p$, and $-3 p)$ showed different occurrences in LMS versus MFH. If our hypothesis of a common oncogenesis for LMS and MFH is verified, genes important for smooth muscle cell differentiation may be localized in some of these six chromosomal regions.

\section{Materials and Methods}

\section{Tumor Samples}

The 27 frozen samples were obtained from three different pathology laboratories. The diagnosis and grading was established according to the recommendations of the French "Sarcoma Group" of the FNCLCC (Fédération Nationale des Centres de Lutte Contre le Cancer). The 27 tumors were from 27 patients. Two of the tumors were from relapses, and 25 from primary tumors. Six tumors were of grade I, 6 were of grade II, and 15 were of grade III. Six of the samples were obtained after a pretreatment of radiotherapy or chemotherapy. Patients consisted of 18 women and 9 men, and ranged in age from 24 to 88 years, mean age of 56.5 years. The main clinical and pathology data are listed in Table 1.

\section{CGH and Image Analysis}

CGH was performed as previously described (Kallionemi et al, 1992). Tumor DNA was directly labeled by nick-translation using an FITC-dUTP nucleotide (DuPont NEN, Boston, Massachusetts). The nick- translation conditions were adjusted to obtain DNA fragments ranging from 500 to $2000 \mathrm{bp}$. SpectrumRed normal male reference DNA (Vysis, Downers Grove, Illinois) was used as control DNA. Four hundred nanograms of tumor DNA, 400 ng of control DNA, and 80 $\mu \mathrm{g}$ of Cot-1 DNA (GibcoBRL, Life Technologies, Gaithersburg, Maryland) were mixed in $15 \mu \mathrm{l}$ of hybridization buffer (50\% formamide, $40 \mathrm{~mm} \mathrm{NaH}_{2} \mathrm{PO}_{4}$, $0.1 \%$ SDS, $10 \%$ dextran sulfate, $2 x$ SSC). CGH pictures from a mean of 10 metaphases were captured using a Leica DMRB fluorescence microscope and a Photometrics Nu200 CCD camera, and analyzed with the Quips XL software (Vysis).

Green to red ratios greater than 1.2 and less than 0.8 were regarded as gains and losses, respectively. Ratios greater than 1.5 were regarded as high level amplifications. Negative controls corresponding to normal DNA hybridized to the same batches of metaphase spreads were included in each series of experiments. Only experiments fulfilling previously defined quality criteria were included (Kallionemi et al, 1994). The analysis of the profiles observed in some GC-rich chromosome regions, such as $1 p, 19$, and 22 , known to give some false-positive results, were interpreted with caution, particularly for the low level of imbalances.

\section{Immunohistochemistry}

Tissue sections were obtained from paraffin blocks. They were deparaffinized in xylene, and rehydrated in a series of ethanol baths. For antigen retrieval, slides were incubated in $10 \mathrm{mM}$ citrate buffer, $\mathrm{pH} 6.1$, for 20 minutes in a microwave oven. Antibodies used to characterize the tumors were purchased from Dako Corporation (Carpinteria, California). The antibodies used were common-muscle actin HHF-35 (Dako M 0635), sarcomeric actin (Dako M 0874), smooth muscle actin (Dako M 0851), desmin (Dako M 0760), vimentin (Dako M 0725), CD 34 (Dako M 7165), and

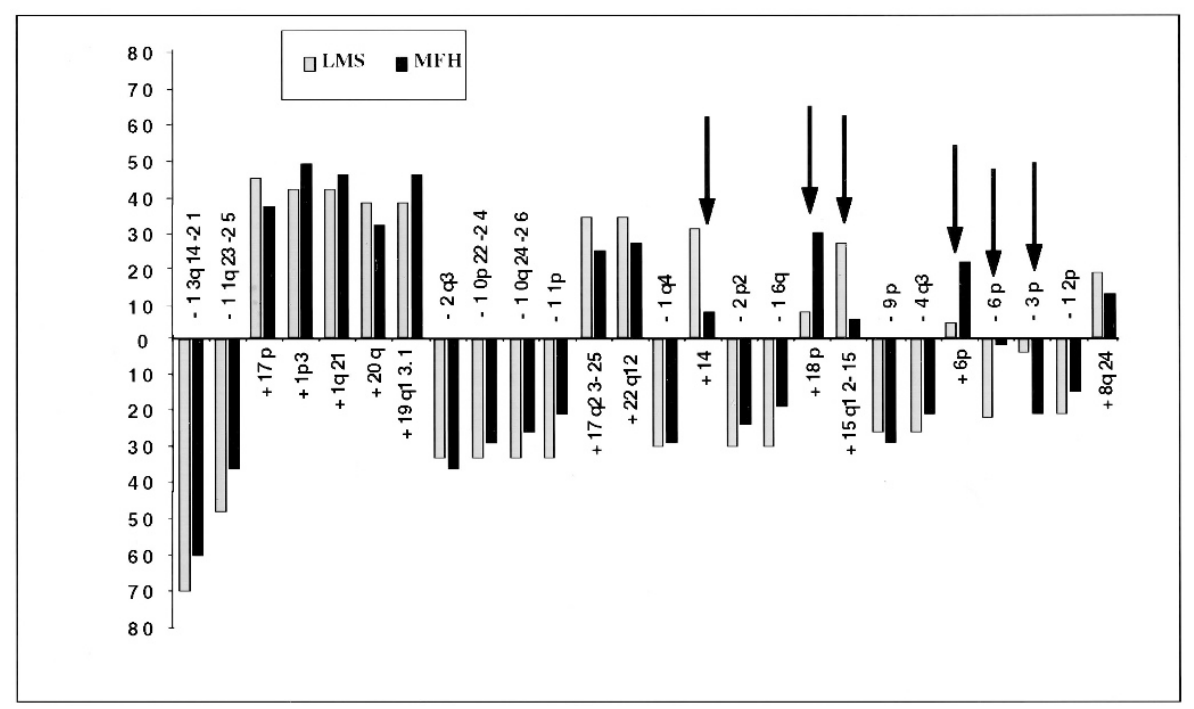

Figure 2.

Comparative frequencies of recurrent imbalances between leiomyosarcoma and malignant fibrous histiocytoma. The six main differences are indicated by arrows. 
CD 117 (c-kit oncoprotein, Dako A 4502). Rb1 1F8, a mouse monoclonal antibody against RB1 protein was purchased from Zymed (San Francisco, California) and used at a dilution of 1:50. Immunohistochemical reactions were performed with an Avidin-Biotin peroxidase complex (Vectastain Elite ABC, Vector, Burlingame, California), using a biotinylated goat anti-mouse secondary antibody and diaminobenzidine as a chromogen. Normal tissue known to express the Rb1-encoded protein was used as a positive control. The original histologic and immunohistochemical slides were not used for the current study and all tissue samples were reprocessed in the same laboratory at the Institut Curie, Paris, France.

\section{References}

Breiner JA, Meis-Kindblom J, Kindblom LG, McComb E, Liu $\mathrm{J}$, Nelson M, and Bridge JA (2000). Loss of $14 q$ and $22 q$ in gastrointestinal stromal tumors (pacemaker cell tumors). Cancer Genet Cytogenet 120:111-116.

Chibon F, Mairal A, Fréneaux $P$, Terrier $P$, Coindre JM, Sastre $X$, and Aurias A (2000). The RB1 gene is the target of chromosome 13 deletions in malignant fibrous histiocytoma. Cancer Res 60:6339-6345.

Costa MJ and Weiss SW (1990). Angiomatoid malignant fibrous histiocytoma: A follow-up study of 108 cases with evaluation of possible histologic predictors of outcome. Am J Surg Pathol 14:1126-1132.

Dehner LP (1988). Malignant fibrous histiocytoma: Nonspecific morphologic pattern, specific pathologic entity, or both? Arch Pathol Lab Med 112:236-237.

Derré J, Lagacé R, Terrier P, Sastre X, and Aurias A (In press, 2000). Constant DNA losses on the short arm of chromosome 1 in a series of malignant gastrointestinal stromal tumors. Cancer Genet Cytogenet.

Dunkel IJ, Gerald WL, Rosenfield NS, Strong EW, Abramson $\mathrm{DH}$, and Ghavimi F (1998). Outcome of patients with a history of bilateral retinoblastoma treated for a second malignancy: The Memorial Sloan-Kettering experience. Med Pediatr Oncol 30:59-62.

El-Rifai W, Sarlomo-Rikala M, Andersson LC, Knuutila S, and Miettinen M (2000). DNA sequence copy number changes in gastrointestinal stromal tumors: Tumor progression and prognostic significance. Cancer Res 60:3899-3903.

El-Rifai W, Sarlomo-Rikala M, Knuutila S, and Miettinen M (1998). DNA copy number changes in development and progression in leiomyosarcomas of soft tissues. Am J Pathol 153:985-990.

El-Rifai W, Sarlomo-Rikala M, Miettinen M, Knuutila S, and Andersson LC (1996). DNA copy number losses in chromosome 14: An early change in gastrointestinal stromal tumors. Cancer Res 56:3230-3233.

Enzinger FM and Weiss SW (1995). Leiomyosarcoma. In: Enzinger FM and Weiss SW, editors. Soft tissue tumors, 3rd ed. St Louis: Mosby, 491-510.

Fanburg-Smith JC and Miettinen M (1999). Angiomatoid malignant fibrous histiocytoma: A clinicopathologic study of 158 cases and further exploration of the myoid phenotype. Hum Pathol 30:1336-1343.

Fletcher CD (1987). Malignant fibrous histiocytoma? Histopathology 11:433-437.
Guccion JG and Enzinger FM (1972). Malignant giant cell tumor of soft parts. An analysis of 32 cases. Cancer 29: 1518-1529.

Hollowood K and Fletcher CD (1995). Malignant fibrous histiocytoma: Morphologic pattern or pathologic entity? Semin Diagn Pathol 12:210-220.

Kallionemi A, Kallionemi OP, Sudar D, Rutovitz D, Gray JW, Waldman F, and Pinkel D (1992). Comparative genomic hybridization for molecular cytogenetic analysis of solid tumors. Science 258:818-821.

Kallionemi OP, Kallionemi A, Piper J, Isola J, Waldman FM, Gray JW, and Pinkel D (1994). Optimizing comparative genomic hybridization for analysis of DNA sequence copy number changes in solid tumors. Genes Chromosom Cancer 10:231-243.

Mairal A, Terrier P, Chibon F, Sastre X, Lecesne A, and Aurias A (1999). Loss of chromosome 13 is the most frequent genomic imbalance in malignant fibrous histiocytomas. A comparative genomic hybridization analysis of a series of 30 cases. Cancer Genet Cytogenet 111:134-138.

Mandahl N, Fletcher CD, Dal Cin P, De Wever I, Mertens F, Mitelman F, Rosai J, Rydholm A, Sciot R, Tallini G, Van den Berghe H, Vanni R, and Willen H (2000). Comparative cytogenetic study of spindle cell and pleomorphic leiomyosarcomas of soft tissues: A report of the CHAMP study group. Cancer Genet Cytogenet 116:66-73.

Merchant W, Calonje E, and Fletcher CD (1995). Inflammatory leiomyosarcoma: A morphological subgroup within the heterogeneous family of so-called inflammatory malignant fibrous histiocytoma. Histopathology 27:525-532.

Miettinen M, Sarlomo-Rikala M, and Lasota J (1999). Gastrointestinal stromal tumors: Recent advances in understanding of their biology. Hum Pathol 30:1213-1220.

Otano-Joos M, Mechtersheimer G, OhI S, Lehnert T, Willeke F, Moller P, Otto HF, Lichter P, and Joos S (1998). Analysis of chromosome copy number changes in leiomyosarcoma through molecular cytogenetic methods. Verh Dtsch Ges Pathol 82:207-209.

Packenham JP, du Manoir S, Schrock E, Risinger JI, Dixon D, Denz DN, Evans JA, Berchuck A, Barrett JC, Devereux TR, and Ried T (1997). Analysis of genetic alterations in uterine leiomyomas and leiomyosarcomas by comparative genomic hybridization. Mol Carcinog 19:273-279.

Parente F, Grosgeorge J, Coindre J-M, Terrier P, Vilain O, and Turc-Carel C (1999). Comparative genomic hybridization reveals novel chromosome deletions in 90 primary soft tissue tumors. Cancer Genet Cytogenet 115:89-95.

Sircar K, Hewlett BR, Huizinga JD, Chorneyko K, Berezin I, and Riddell RH (1999). Interstitial cells of Cajal as precursors of gastrointestinal stromal tumors. Am J Surg Pathol 23:377389.

Suijkerbuijk RF, Olde Weghuis DE, Van den Berg M, Pedeutour F, Forus A, Myklebost O, Glier C, Turc-Carel C, and Geurts van Kessel A (1994). Comparative genomic hybridization as a tool to define two distinct chromosome 12-derived amplification units in well-differentiated liposarcomas. Genes Chromosom Cancer 9:292-295.

Weiss SW and Enzinger FM (1977). Myxoid variant of malignant fibrous histiocytoma. Cancer 39:1672-1685. 